\title{
A Longitudinal Study of Cerebral Blood Flow Over the First 30 Months
}

\author{
MARTIN KEHRER AND MARTIN SCHÖNING
}

Department of Paediatric Neurology and Developmental Medicine, University Children's Hospital, Tübingen 72076, Germany

\begin{abstract}
To investigate prospectively the development of cerebral perfusion during infancy, serial quantitative measurements of cerebral blood flow (CBF) volume were performed in two healthy children from birth up to the age of 30 mo. A total of $28 \mathrm{CBF}$ volume measurements were done in either of the children. Absolute flows were measured in the internal carotid and vertebral arteries on both sides. Blood flow was calculated as the product of angle-corrected time-averaged flow velocity and the cross-sectional area of the vessel. Starting from 67 and $80 \mathrm{~mL} / \mathrm{min}$, respectively, at birth an almost 10-fold increase of CBF volume was observed in both children during the examination period. Half of this rise occurred during the first 6 mo, probably reflecting the steep metabolic incline during this period of synaptogenesis. The continuous increase in CBF volume after the sixth month of life mainly corresponds to brain growth. Estimated CBF (based on estimated brain weights) increased from 21 and $23 \mathrm{~mL} 100 \mathrm{~g}^{-1} \mathrm{~min}^{-1}$, respectively, after birth to 46 and $53 \mathrm{~mL} 100 \mathrm{~g}^{-1} \mathrm{~min}^{-1}$, respectively, during the first 6 mo of life in both children, remaining stable thereafter. This study is the first to provide longitudinal data of $\mathrm{CBF}$ during the first 30 mo after birth. (Pediatr Res 66: 560-564, 2009)
\end{abstract}

$\mathrm{C}$ erebral blood flow (CBF) volume can be measured (in milliliter per minute) with the use of sonographic flowmetry of the extracranial brain-feeding arteries $(1,2)$. The method is noninvasive and innocuous and therefore suited for serial measurements, which has been shown in adult neurointensive care patients $(3,4)$ and in a longitudinal study in preterm infants (5). The accuracy of the measurement depends on a motionless position of the patient and a strict compliance with a standardized study protocol. In a first interobserver reproducibility performed in children and adults, a $10.0 \%$ coefficient of variation has been found (6). The result was comparable with that of a reproducibility study in adult men where two subsequent $\mathrm{H}_{2}{ }^{15} \mathrm{O}$-PET scans had been done (7). A recent interobserver reproducibility study performed in neonates resulted in a coefficient of variation of only $6.3 \%$ (8).

Reference data for CBF volume are available for preterm and term neonates (gestational age, 32-42 wk) (9) and for children from 3 y up to the age of 18 y (10). Although the CBF volume in term neonates is about $70 \mathrm{~mL} / \mathrm{min}(9)$, it is 10 times higher in 3-y-old children (about $700 \mathrm{~mL} / \mathrm{min}$ ) (10). The dynamics of $\mathrm{CBF}$ volume increase, however, from birth up to

Received March 26, 2009; accepted July 14, 2009.

Correspondence: Martin Kehrer, M.D., Department of Paediatric Neurology and Developmental Medicine, University Children's Hospital, Hoppe-Seyler-Strasse 1,Tübingen 72076, Germany; e-mail: martin.kehrer@med.uni-tuebingen.de

Supported by grant from the Beitlich Foundation. the age of $3 \mathrm{y}$ is still unclear. The aim of the presented longitudinal study was to describe the development of cerebral perfusion during the first 30 mo of life by serial measurements of CBF volume in two healthy children.

\section{METHODS}

Probands. Two healthy, term born children [1 female ("F"), 1 male ("M")] were involved in a prospective study. Birth weights were $3210 \mathrm{~g}$ (M) and $2810 \mathrm{~g}(\mathrm{~F})$, body lengths at birth were $53 \mathrm{~cm}(\mathrm{M})$ and $50 \mathrm{~cm}(\mathrm{~F})$, and head circumferences at birth were $34.0 \mathrm{~cm}(\mathrm{M})$ and $33.0 \mathrm{~cm}(\mathrm{~F})$. Cerebral B-mode ultrasound examinations performed until the closure of the anterior fontanel were always normal in both children. Neither of them ever presented cardiologic or neurologic abnormalities or received any specific medication. Motor, language, cognitive, and emotional development during the first $3 \mathrm{y}$ of live was normal. At the age of $30 \mathrm{mo}$, Bayley Scales for Infant Development-II (11) were performed in both children; the mean developmental quotient in either of the two children was 111 . The Kaufman Assessment Battery for Children (12) showed an intelligence quotient of 103 at the age of 5 y 7 mo (F) and an intelligence quotient of 113 at the age of 5 y 6 mo (M), respectively. Informed consent was given by the parents in both cases. The study was approved by the Ethical Committee of the University of Tübingen.

Study protocol. Color duplex sonography of all the extracranial cerebral arteries, i.e., the internal carotid artery (ICA) and vertebral artery (VA) of both sides, was performed over the first $2 \frac{1}{2}$ y of life according to the following schedule. A total of 28 measurements of CBF volume were performed in either of the two children: one measurement in each of the first 4 wk of life $(n=4)$; one measurement every $2 \mathrm{wk}$ from the second to the sixth month of life $(n=10)$; one measurement every month from the seventh to the twelfth month of life $(n=6)$; one measurement every 2 mo during the second year of life $(n=6)$; one measurement in the first and in the second quarter of the third year of life, respectively $(n=2)$. All measurements were performed by the same examiner (M.K.).

Examination procedure. All measurements were performed immediately after the children fell asleep in the examination room. The examination of all the extracranial arteries required about $20 \mathrm{~min}$. The subjects lay in a supine position with their heads slightly elevated and turned to the contralateral side by about $10^{\circ}$ during the examination of the VA, and by $25-40^{\circ}$ during the examination of the ICA. All the measurements could be completed successfully.

Measurements were made with a $10.0-\mathrm{MHz}$ linear transducer (aperture size, $26 \mathrm{~mm}$ ) of a computed sonography system (Acuson 128/XP10, Mountain View, CA) at a location at least $1.0 \mathrm{~cm}$ above the bifurcation in the ICA. The VA was examined in the transverse part (V2 segment), generally between the levels $\mathrm{C} 3$ and $\mathrm{C} 5$.

The measurement technique was essentially the same as that described previously in preterm and term neonates $(5,9,13)$ and in children from $3 \mathrm{y}$ of age onward $(10,14)$. The luminal diameter of the vessels, $d$, was measured on a magnified, high-resolution B-mode image between the bright internal layers of the parallel vessel walls at the exact site of color duplex measurement and perpendicular to the longitudinal axis of the artery. The calipers could be adjusted in $0.1-\mathrm{mm}$ increments. The mean of duplicate luminal measurements was calculated for each vessel. The color duplex sonography procedure consisted of acquiring a color Doppler image of the vessel and carrying out an

Abbreviations: CBF, cerebral blood flow; ICA, internal carotid artery; TAV, time-averaged velocity; VA, vertebral artery 
angle-corrected pulsed Doppler time-frequency analysis of the intravascular flow in each artery. At a site of undisturbed color Doppler flow, the pulsed Doppler sample volume was positioned over the entire width of the vessel. Thus, the length of the sampling volume was adjusted to cover from the near to the far-away vessel wall to ensure that the transverse extension of the drop-shaped sample volume of the Doppler beam sufficed to insonate the vessel in a homogenous and representative manner. This is of particular importance in larger vessels to avoid systematic errors. The electronic steering feature of the linear probe was used to keep the angle of insonation as low as possible, in most cases between $30^{\circ}$ and $55^{\circ}$ in the ICA and between $40^{\circ}$ and $60^{\circ}$ in the VA. Visual checks on the maximal luminal width and acoustic checks on the optimum time-frequency Doppler signal served to ensure that the sample volume passed through the center of the vessel. A spectral curve was then recorded with the color Doppler image "frozen." The Doppler signal was evaluated only if it was stable for at least $5 \mathrm{~s}$ and did not contain bidirectional frequencies (which would indicate disturbed flow). The angle of insonation was adjusted in $1^{\circ}$ increments by aligning an angle indicator parallel to the walls of the vessels and along the intravascular color Doppler stream.

Angle-corrected time-averaged flow velocity (TAV), i.e., the integral of the mean flow velocities of all moving particles passing the sample volume, was measured over three to five complete cardiac cycles. In this way, the pulsatile parabolic flow is mathematically transformed into a continuous plug flow. After the measurement of the luminal diameters and TAV of all vessels had been completed, the intravascular flow volume (FV) was determined by calculating the product of the angle-corrected TAV and the cross-sectional area of the circular vessel $\mathrm{A}$ with the formula $\mathrm{FV}=\mathrm{TAV} \times \mathrm{A}=\mathrm{TAV} \times$ $\left[(\mathrm{d} / 2)^{2} \times \pi\right]$. CBF volume was determined by adding the bilateral ICA and VA FVs, i.e., the integral of flow passing through the anterior and posterior brain circulation.

In addition, $\mathrm{CBF}$ volume (in milliliter per minute) was converted into "estimated CBF" [in milliliter per 100 gram brain weight (BrW) per minute]. For this purpose, the $\mathrm{BrW}$ of each child was estimated using an equation based on head circumference measurements (15), which were taken at each examination day (according to the data of Dobbing and Sands (15), BrW in children during the first $2 \mathrm{y}$ of postnatal life is expressed as a function of head circumference $\left.(\mathrm{HC}): \mathrm{BrW}=(\mathrm{HC})^{3} / 100-[3000 /(2 \times \mathrm{HC})]\right)$. "Estimated CBF" was calculated separately for each measurement in either of the two children with the use of the present-day head circumference.

To investigate the physiologic factors that mainly contribute to the dynamic increase in CBF volume during early postnatal brain development (vessel diameter or intravascular flow velocity), we also determined i) the "cross-sectional area of all insonated vessels" (by adding the four crosssectional areas A of the ICA and VA of both sides) and ii) the "mean TAV", i.e., the sum of TAV of the ICA and VA of both sides divided by four.

\section{RESULTS}

In both children, there was a marked increase in CBF volume during the first 6 mo of life. Thereafter, CBF volume continued to rise, but less steeply (Fig. 1).

There was a rise in bilateral ICA FVs and in bilateral VA FVs with increasing age (Table 1). The proportion of VA FV to total CBF volume increased from $27 \%$ (F) and $20 \%(\mathrm{M})$, respectively, after birth to $36 \%$ (F) and $32 \%(\mathrm{M})$, respectively,

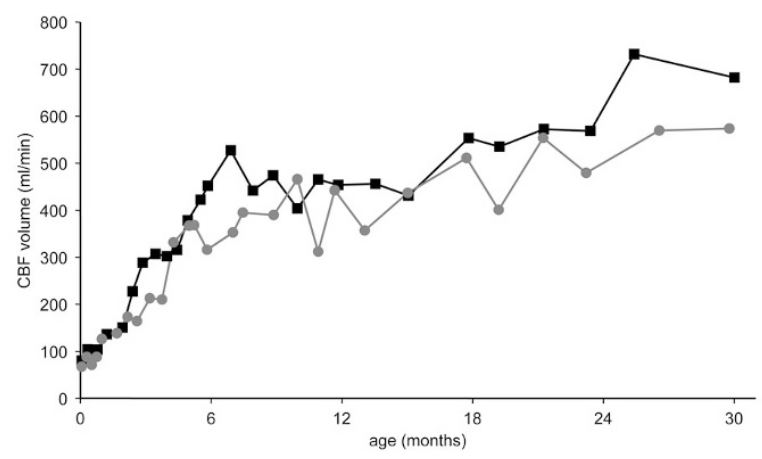

Figure 1. Development of cerebral blood flow volume (CBF volume) from birth to the third year of life in the two probands $(\mathbf{\square}=\mathrm{M}, \bigcirc=\mathrm{F})$. at the age of 6 mo and remained fairly stable at this level until the end of the examination period (Table 1).

The BrW data, as estimated by the method of Dobbing and Sands (15), are displayed in Table 1. In both children, estimated CBF increased from 21 and $23 \mathrm{~mL} 100 \mathrm{~g}^{-1} \mathrm{~min}^{-1}$, respectively, after birth to 46 and $53 \mathrm{~mL} 100 \mathrm{~g}^{-1} \mathrm{~min}^{-1}$, respectively, at the age of 6 mo, remaining stable thereafter until the end of the examination period (Fig. 2).

Data of TAV and luminal diameters of both ICA and VA of both sides are shown in Table 1. The mean TAV of all extracranial arteries doubled during the first 6 mo of life, remaining constant at this level afterward (Fig. 3). By way of contrast, the cross-sectional area of all insonated vessels showed a continuous 5-fold increase during the examination period (Fig. 4).

\section{DISCUSSION}

This study provides first quantitative CBF volume data for the first postnatal years, which are characterized by an enormous spurt in brain growth and development. Sonographic flowmetry of the extracranial brain-feeding arteries is a highly reproducible, innocuous, quantitative measurement method for CBF and suited for longitudinal studies. This method, however, requires training, a quiet infant, and takes about 15 min for a measurement; therefore, rapid changes of blood flow cannot be detected.

The lack of normal values for CBF volume during the first $3 \mathrm{y}$ of life is due to the difficulty in examining children of this age group. On the one hand, cooperation cannot be expected, as is the case with children from 3 y of age onward. Therefore, an examination in an awake resting state is not feasible. On the other hand, spontaneous sleep is much less frequent than in preterm and term neonates. Other quantitative CBF measurements methods like $\mathrm{H}_{2}{ }^{15} \mathrm{O}$-PET or the ${ }^{133}$ Xenon-clearance technique cannot be applied to healthy children for ethical reasons.

In accordance with the normal data of CBF volume for preterm and term neonates (9) and for children from $3 \mathrm{y}$ of age onward (10), we found a rise in CBF volume from 67 and 80 $\mathrm{mL} / \mathrm{min}$, respectively, to 573 and $682 \mathrm{~mL} / \mathrm{min}$, respectively, during the examination period. Remarkably, half of this almost 10-fold increase of CBF volume between birth and the third year of life already occurs during the first 6 mo of life.

To obtain an estimate of $\mathrm{BrW}$ for each examination in either of the two children, we used an equation published by Dobbing and Sands (15). The BrW data obtained in this manner were similar to those provided by anatomical studies on children of comparable age (16-18). When we converted CBF volume (in milliliter per minute) into "estimated CBF" (in milliliter per 100 gram $\mathrm{BrW}$ per minute), we found a clear rise of CBF from about $20 \mathrm{~mL} 100 \mathrm{~g} \mathrm{~g}^{-1} \mathrm{~min}^{-1}$ to $50 \mathrm{~mL} 100 \mathrm{~g}^{-1}$ $\min ^{-1}$ during the first $6 \mathrm{mo}$ of life in both children. Thereafter, $\mathrm{CBF}$ remained constant at $50 \mathrm{~mL} 100 \mathrm{~g}^{-1} \mathrm{~min}^{-1}$.

The marked increase in perfusion intensity during the first half year of life reflects the rising metabolic demand in this period, which may correspond to the concurrent increase in synaptic density (19). Similarly, a marked in- 
Table 1. Data of flow volumes, luminal diameters, and TAV of both ICA and VA of both sides

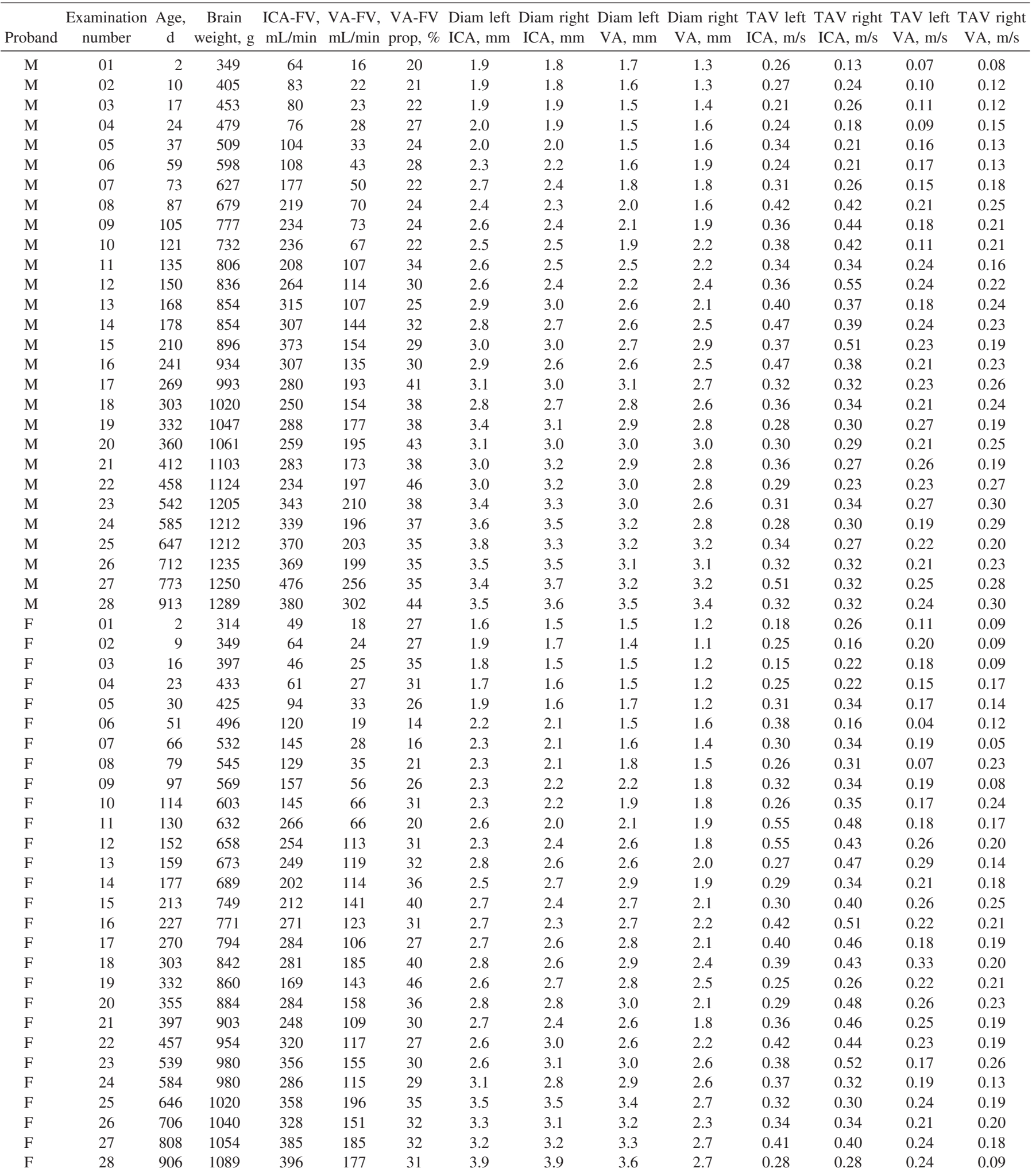

Brain weight, brain weight as estimated by the method of Dobbing and Sands(15); ICA, internal carotid artery; VA, vertebral artery; FV, flow volume; VA-FV prop, proportion of VA flow volume to total CBF volume; Diam, luminal diameter of the vessel; TAV, time-averaged velocity.

crease in local cerebral metabolic rates for glucose during the first postnatal years has been shown by PET studies (20). However, although the rise in CBF volume after the sixth month of life mainly corresponds to brain growth, local cerebral metabolic rates for glucose continuously increase during the first $3 \mathrm{y}$ of life. The combination of these two findings could indicate an improving efficiency of the brain with respect to the extraction of glucose from the blood after the sixth month of life. This assumption would be in contrast to the results of a study using the $\mathrm{N}_{2} \mathrm{O}$ 


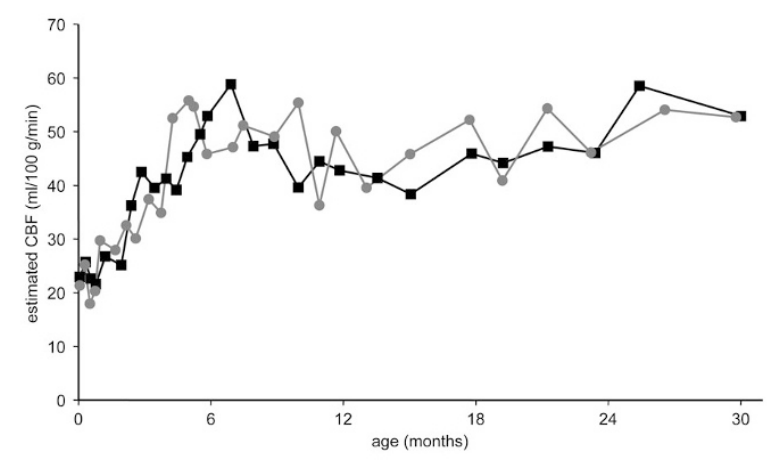

Figure 2. Development of estimated cerebral blood flow (estimated CBF) from birth to the third year of life in the two probands $(\mathbf{\square}=\mathrm{M}, \quad=\mathrm{F})$.

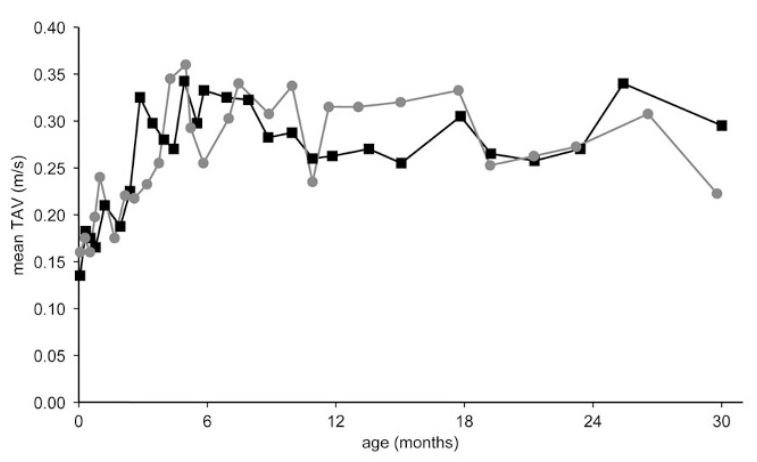

Figure 3. Development of the mean time-averaged flow velocity (TAV) of both ICA and VA of both sides from birth to the third year of life in the two probands $(\square=\mathrm{M}, \mathrm{\square}=\mathrm{F})$.

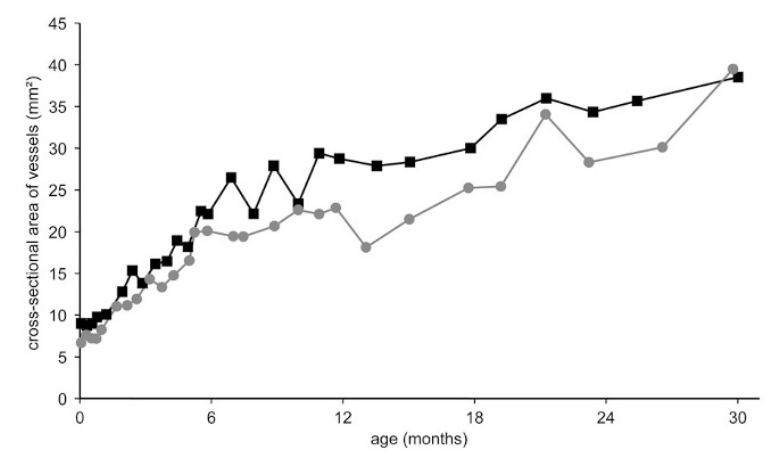

Figure 4. Development of the cross-sectional area of all insonated vessels (both ICA and VA of both sides) from birth to the third year of life in the two probands $(\mathbf{\square}=\mathrm{M}, \mathrm{O}=\mathrm{F})$.

technique, which did not show any correlation of CBF or cerebral uptake of glucose to age in anesthetized children (21).

The values of CBF volume that we found in the first week of life in the two children $(67$ and $80 \mathrm{~mL} / \mathrm{min}$, respectively, corresponding to 21 and $23 \mathrm{~mL} 100 \mathrm{~g}^{-1} \mathrm{~min}^{-1}$, respectively) are in line with the normal data for term neonates (9). A CBF volume of 573 and $682 \mathrm{~mL} / \mathrm{min}$ (corresponding to $53 \mathrm{~mL} / 100$ $\mathrm{g}$ min in both children), which we found at the end of the examination period, fits well to the normal average values in children at 3 y of age (10).

Color duplex flowmetry of CBF volume cannot be used to determine regional CBF. However, it is possible to measure the relative contributions of the anterior and the posterior brain circulation to total CBF volume. The mean contribution of the posterior cerebral circulation to total CBF volume increased from 20 to $27 \%$ after birth to 27 to $46 \%$ beyond the sixth month of life. Previous studies showed a constant proportion of the posterior cerebral circulation to total CBF volume of $26 \%$ in preterm and term neonates (9) and a drop from $31 \%$ in children 3 y of age to $24 \%$ in 18 -y olds, then remaining constant at $24 \%$ in adults $(10,22)$. Thus, the findings in these two children fit well to the corresponding data at birth and the third year of life, on the basis of which an increase of the proportion of the posterior cerebral circulation to total CBF volume from 26 to $31 \%$ had to be expected. The highest values $(40-45 \%)$ were found at the end of the first year of life. In this period of life, locomotion and coordination are developing rapidly. These are main tasks of brain areas (e.g., the visual cortex and the cerebellum), which are supplied by the posterior cerebral circulation.

Regarding the hemodynamic data in detail, the increase in $\mathrm{CBF}$ volume during the first $3 \mathrm{y}$ of life is due to a continuous rise of the cross-sectional area of the vessels, and the remarkable intensification during the first 6 mo of life is achieved by an additional increase in blood flow velocity.

During the neonatal period, the FVs, flow velocities, and vessel diameters in the two children were within the ranges of the previously established normal data in neonates $(9,13)$. At the end of the examination period $\left(2 \frac{1}{2} \mathrm{y}\right.$ of age $)$, the respective data of FVs, flow velocities, and vessel diameters lie within (or just at the lower limit of) the normal range of values of children aged $3-10$ y $(10,14)$. Normal motor and cognitive development in the children was documented.

Thus, this longitudinal study performed in two healthy children, although not providing a normative database, allows to outline for the first time the development of cerebral perfusion and hemodynamics during early postnatal brain development.

\section{REFERENCES}

1. Schöning M, Walter J, Scheel P 1994 Estimation of cerebral blood flow through color duplex sonography of the carotid and vertebral arteries in healthy adults. Stroke 25:17-22

2. Kehrer M, Goelz R, Krägeloh-Mann I, Schöning M 2002 Measurement of volume of cerebral blood flow in healthy preterm and term neonates with ultrasound. Lancet 360:1749-1750

3. Schöning M, Scheel P, Holzer M, Fretschner R, Will BE 2002 Can a critical lower threshold of cerebral perfusion be determined? A pilot observational study. Neurology 58:1860-1861

4. Schöning M, Scheel P, Holzer M, Fretschner R, Will BE 2005 Volume measurement of cerebral blood flow: assessment of cerebral circulatory arrest. Transplantation 80:326-331

5. Kehrer M, Blumenstock G, Ehehalt S, Goelz R, Poets C, Schöning M 2005 Development of cerebral blood flow volume in preterm neonates during the first two weeks of life. Pediatr Res 58:927-930

6. Schöning M, Scheel P 1996 Color duplex measurement of cerebral blood flow volume: intra- and interobserver reproducibility and habituation to serial measurements in normal subjects. J Cereb Blood Flow Metab 16:523-531

7. Matthew E, Andreason P, Carson RE, Herscovitch P, Pettigrew K, Cohen R, King C, Johanson CE, Paul SM 1993 Reproducibility of resting cerebral blood flow measurements with $\mathrm{H} 2(15) \mathrm{O}$ positron emission tomography in humans. J Cereb Blood Flow Metab 13:748-754

8. Ehehalt S, Kehrer M, Goelz R, Poets C, Schöning M 2005 Cerebral blood flow volume measurements with ultrasound: interobserver reproducibility in preterm and term neonates. Ultrasound Med Biol 31:191-196 
9. Kehrer M, Krägeloh-Mann I, Goelz R, Schöning M 2003 The development of cerebral perfusion in healthy preterm and term neonates. Neuropediatrics 34:281-286

10. Schöning M, Hartig B 1996 Age dependence of total cerebral blood flow volume from childhood to adulthood. J Cereb Blood Flow Metab 16:827-833

11. Bayley N 1993 Bayley Scales of Infant Development (BSID-II). 2nd ed. Psychological Corporation, San Antonio, TX

12. Melchers P, Preuss U 1994 Kaufman Assessment Battery for Children K-ABC, Deutschsprachige Fassung. Swets, Frankfurt, Germany

13. Kehrer M, Goelz R, Schöning M 2004 The development of haemodynamics in the extracranial cerebral arteries of healthy preterm and term neonates. Ultrasound Med Biol 30:283-287

14. Schöning M, Hartig B 1998 The development of hemodynamics in the extracranial carotid and vertebral arteries. Ultrasound Med Biol 24:655-662

15. Dobbing J, Sands J 1978 Head circumference, biparietal diameter and brain growth in fetal and postnatal life. Early Hum Dev 2:81-87
16. Dekaban AS, Sadowsky D 1978 Changes in brain weights during the span of human life: relation of brain weights to body heights and body weights. Ann Neurol 4:345-356

17. Dobbing J, Sands J 1973 Quantitative growth and development of human brain. Arch Dis Child 48:757-767

18. McLennan JE, Gilles FH, Neff RK 1983 A model of growth of the human fetal brain In: Gilles FH, Leviton A, Dooling EC (eds) The Developing Human Brain: Growth and Epidemiologic Neuropathology. John Wright-PSG, Boston, MA, pp 43-58

19. Huttenlocher PR 1990 Morphometric study of human cerebral cortex development Neuropsychologia 28:517-527

20. Chugani HT, Phelps ME, Mazziotta JC 1987 Positron emission tomography study of human brain functional development. Ann Neurol 22:487-497

21. Settergren G, Lindblad BS, Persson B 1980 Cerebral blood flow and exchange of oxygen, glucose, ketone bodies, lactate, pyruvate and amino acids in anesthetized children. Acta Paediatr Scand 69:457-465

22. Scheel P, Ruge C, Petruch UR, Schöning M 2000 Color duplex measurement of cerebral blood flow volume in healthy adults. Stroke 31:147-150 